\title{
Shopping Behavior for ADHD Drugs: Results of a Cohort Study in a Pharmacy Database
}

\author{
M. Soledad Cepeda $\cdot$ Daniel Fife $\cdot$ Joris Berwaerts • \\ Yingli Yuan · Greg Mastrogiovanni
}

Published online: 14 August 2014

(C) The Author(s) 2014. This article is published with open access at Springerlink.com

\begin{abstract}
Objective Attention-deficit hyperactivity disorder (ADHD) medications are subject to abuse, misuse, and diversion. Obtaining ADHD prescriptions from multiple prescribers or filled across multiple pharmacies, known as 'doctor shopping', may reflect such unsanctioned use. We sought to create a definition of shopping behavior that differentiated ADHD medications from medications with low risk of diversion, i.e. asthma medications, and describe the incidence, frequency, and demography of shopping behavior.

Methods This was a retrospective cohort study in a pharmacy database-LRx - covering $65 \%$ of US retail pharmacies. Subjects had ADHD or asthma medication dispensed between February 2011 and January 2012. We followed subjects for 18 months to assess the number with overlapping dispensings from different prescribers, and the number of prescribers and pharmacies involved in those dispensings.

Results We included 4,402,464 subjects who were dispensed ADHD medications, and 6,128,025 subjects who were dispensed asthma medications. Overlapping prescriptions from two or more prescribers dispensed by three or more pharmacies was four times more frequent in the ADHD cohort than in the asthma cohort. Using this definition, ADHD medication shopping behavior was more common among experienced users than naïve users, and
\end{abstract}

M. S. Cepeda $(\square) \cdot$ D. Fife $\cdot$ J. Berwaerts

Janssen Research \& Development, LLC, 1125 Trenton

Harbourton Rd, Titusville, NJ 08560, USA

e-mail: scepeda@its.jnj.com

Y. Yuan · G. Mastrogiovanni

IMS Health, Collegeville, PA, USA was most common in subjects aged 10-39 years. Among subjects who shopped, $57.4 \%$ shopped only once (accounting for $22.4 \%$ of episodes), and $9.2 \%$ shopped six or more times (accounting for $42.0 \%$ of episodes). Shoppers more often received stimulant ADHD drugs than non-stimulants.

Conclusions Overlapping prescriptions by different prescribers and filled at three or more pharmacies defines ADHD medication shopping. Shopping behavior is most common in adolescents and younger adults. A small proportion of shoppers is responsible for a large number of shopping episodes.

\section{Key Points}

Attention-deficit hyperactivity disorder (ADHD) medications may be subject to abuse, misuse, and diversion.

We found that overlapping prescriptions from two or more prescribers dispensed by three or more pharmacies defines ADHD medication shopping.

\section{Introduction}

Medications for the treatment of attention-deficit hyperactivity disorder (ADHD) are subject to misuse, abuse, and diversion [1-3]. The non-medical use of ADHD medications in high-school-age children in the US is estimated at around $9 \%$, and in college-age individuals goes from 5 to $35 \%$ [1]. The increase in recent years in the number of emergency visits involving ADHD medications for non- 
medical use (use not prescribed for the respondent by a physician or use only for the experience or feeling caused [4]) [5] reflects these problems. Obtaining ADHD prescriptions from multiple prescribers and/or filling prescriptions across multiple pharmacies, often called doctor or pharmacy shopping, may reflect such unsanctioned use $[1,6]$.

Doctor-shopping behavior is increasingly recognized for opioids [7-10], but less is known about doctor-shopping behavior for ADHD medications. On the basis of data from California's Prescription Monitoring Programs, $1.4 \%$ of subjects dispensed ADHD medication had multiple providers in 2007 [11]. However, it is unclear how the behavior should be defined, how many individuals engage in the behavior (incidence), how often it occurs in a given subject or across subjects (frequency), the impact of age and sex on such behavior, or whether a small proportion of subjects are responsible for a large proportion of shopping episodes (concentration).

Developing a definition of shopping behavior for ADHD medications will help identify subjects who may be at higher risk of misusing/abusing or diverting. We sought to create an operational definition of shopping behavior that differentiated ADHD medications from medications with low risk of abuse or diversion, such as asthma medications. Such a definition will therefore decrease the risk of inappropriately identifying individuals with legitimate use of ADHD medications as being at increased risk of misusing/ abusing or diverting those medicines. Such misclassification may adversely affect the individual who is misclassified (e.g. social stigma) and, secondly, it is potentially deleterious to research studies that assess shopping behavior and abuse because random misclassification would bias the studies toward the null and thus obscure the signals of interest. Once we defined shopping behavior, we also sought to describe its incidence and frequency, the impact of age and sex on shopping behavior, and the type of ADHD medication involved in the shopping episodes.

\section{Method}

\subsection{Data Collection}

We conducted a population-based retrospective cohort study using the LRx database, a longitudinal pharmacy database that covers $65 \%$ of all retail dispensing in the US and includes all types of pharmacies-chains, food stores, mass merchandisers, and independent stores. From each of the pharmacies in the panel, the database captures all prescriptions that were dispensed, regardless of payment type. In particular, it includes prescriptions filled for patients with any insurance type (commercial, Medicare, Medicaid) and prescriptions paid for entirely in cash. Dispensing records are collected directly from pharmacies, which provide encrypted patient identifiers compliant with Health Insurance Portability and Accountability Act (HIPAA) privacy regulations. The LRx database includes data on the subject (de-identified), the pharmacy, and the prescriber.

To uniquely identify a subject who filled prescriptions at multiple pharmacies, a probabilistic multi-level match was performed using a proprietary algorithm based on encrypted non-identifiable data elements including sex, date of birth, last name, first name, address, city, state, zip code, and payer identification.

\subsection{Inclusion Criteria}

We included all subjects dispensed an ADHD or asthma medication between 1 February 2011 and 31 January 2012 who had data available for at least 4 months prior to the first dispensing (index date), and whose pharmacies consistently supplied data to the LRx database during the entire study period. Each subject was followed for 18 months from his/her index date.

A subject who was dispensed ADHD and asthma medications could be a member of both cohorts.

\subsection{Prescription/Dispensing Data}

We included all ADHD medications whose ingredients were approved by the US FDA for the treatment of ADHD. These were the stimulants amphetamine, dexmethylphenidate, dextroamphetamine, lisdexamfetamine, methamphetamine, and methylphenidate, and the non-stimulants atomoxetine, clonidine, and guanfacine.

The asthma medications included were inhaled bronchodilators, inhaled steroids, inhaled steroid/long-acting $\beta$ agonist combinations, and oral leukotriene inhibitors. Asthma medications were used as a comparator because they are frequently used by a population with roughly similar demographic characteristics as the population using ADHD medications [12], including a large representation of children and young adults, and are not believed to be widely abused or diverted [13].

Subjects who were not dispensed any ADHD medication during the 4 months before their index date were considered 'naive'. The 4-month period, rather than a shorter period, was adopted to decrease the risk of misclassifying as naïve a subject who was receiving an ADHD medication during the school year but took a planned break in its use during 3 or 4 months of vacation (i.e. took a 'drug holiday'). 


\subsection{Outcome}

We assessed the number of subjects with overlapping dispensings of medications prescribed by different prescribers, and the number of prescribers and number of pharmacies involved in those dispensings, during the 18 months of follow-up. For subjects with more than one event of multiple overlapping filled prescriptions, we selected the one event with the maximum number of overlapping prescriptions. Note that a prescriber can write more than one prescription for a given individual, therefore the total number of pharmacies making dispensings for that individual may exceed the number of prescribers.

An overlap occurred when two or more dispensings of medications prescribed by different prescribers were active on the same day (i.e. a medication was dispensed during the days' supply of another dispensed medication). The overlapping dispensings could be for the same or different ADHD or asthma medications.

To set a definition of shopping behavior that differentiated ADHD medications from asthma medications, we qualitatively compared the frequency distribution of subjects across the number of prescribers and pharmacies between the two cohorts-the larger the ratio in the proportion of subjects, the larger the difference in behavior between the two cohorts. The best cut-off of number of pharmacies and number of prescribers also had to have a sufficient proportion of subjects to provide a useful marker of unsanctioned use.

Once the definition was selected, we identified subjects who met the definition, i.e. subjects with at least one event of overlapping prescriptions written by two or more prescribers and filled at three or more pharmacies. The index or qualifying event did not necessarily occur during the episode with the highest number of overlapping prescriptions. We then assessed how soon the shopping episode was observed during follow-up of a given subject (i.e. median time from index date to first shopping episode), the total number of events across all subjects according to age category, sex, and prior exposure (naïve or experienced), and the concentration of shopping (extent to which a relatively small proportion of shoppers accounted for a relatively large proportion of shopping episodes). Each time there was a new dispensing, the definition of shopping behavior was applied and, if the criteria were met, a new shopping episode was counted.

To make sure that the subjects dispensed prescribed asthma medication had a similar age distribution to the subjects dispensed ADHD medications, the asthma subjects were frequency-matched to the ADHD subjects by single year of birth.

This study used completely anonymized data and did not involve patient contact. The New England Institutional
Review Board determined that this was not human-subject research.

\section{Results}

A total of 4,402,464 subjects dispensed ADHD medications and 6,128,025 subjects dispensed asthma medications were included in the analysis. The age distribution (mean $\pm \mathrm{SD}$ ) of the subjects was similar in the two cohorts-24.1 \pm 16.2 years of age in the ADHD medication cohort and $24.2 \pm 16.8$ in the asthma medication cohort, as would be expected from the age matching. In the ADHD medication cohort, $43.9 \%$ were female, and in the asthma medication cohort, $55.6 \%$ were female.

The distribution of pharmacies and prescribers visited by subjects was markedly different in subjects who received ADHD drugs compared with those who received asthma drugs.

Overlapping prescriptions written by two or more prescribers and dispensed at two or more pharmacies were approximately twofold more frequent in the ADHD medication cohort than in the asthma medication cohort, and occurred in 198,923 subjects in the ADHD medication cohort $(4.5 \%)$ and in 120,163 subjects in the asthma medication cohort $(2.0 \%)$ [Tables 1 and 2].

Overlapping prescriptions written by two or more prescribers and dispensed at three or more pharmacies were approximately fourfold more frequent in the ADHD medication cohort than in the asthma medication cohort, and occurred in 11,861 subjects in the ADHD medication cohort $(0.27 \%)$ and in 4,226 subjects in the asthma medication cohort $(0.07 \%)$ [Tables 1 and 2].

Overlapping prescriptions written by two or more prescribers and dispensed at five or more pharmacies were approximately 28 -fold more frequent in the ADHD medication cohort than in the asthma medication cohort; however, this occurred in only 143 subjects in the ADHD medication cohort $(0.003 \%)$, and in seven subjects in the asthma medication cohort $(0.0001 \%)$ [Tables 1 and 2].

We therefore adopted overlapping prescriptions written by two or more prescribers and filled at three or more pharmacies as the definition of ADHD medication shopping behavior. This definition of shopping behavior clearly discriminated between subjects dispensed ADHD medications and subjects dispensed asthma medications, and was sufficiently common to serve as a marker suggestive of unsanctioned use.

Using this definition, we found that ADHD medication shopping behavior was most commonly observed between 10 and 39 years of age. No large differences in frequency of shopping behavior were observed between men and women. Shopping behavior was observed in 13,707 
Table 1 Number of subjects exposed to ADHD medications, with their number of prescribers and pharmacies visited ${ }^{\mathrm{a}}$

\begin{tabular}{lllllllll}
\hline Number of pharmacies & 1 & 2 & 3 & 4 & 5 & 6 & 7 & Total \\
\hline Number of prescribers & & & & & & & \\
1 & $3,555,122(80.8)$ & $(0)$ & $(0)$ & $(0)$ & $(0)$ & $(0)$ & $(0)$ & $3,555,122(80.8)$ \\
2 & $640,049(14.5)$ & $182,818(4.2)$ & $9,056(0.2)$ & $611(0)$ & $44(0)$ & $8(0)$ & $2(0)$ & $832,588(18.9)$ \\
3 & $8,183(0.2)$ & $4,141(0.1)$ & $1,616(0)$ & $278(0)$ & $48(0)$ & $4(0)$ & $1(0)$ & $14,271(0.3)$ \\
4 & $184(0)$ & $100(0)$ & $88(0)$ & $57(0)$ & $20(0)$ & $3(0)$ & $(0)$ & $452(0)$ \\
5 & $3(0)$ & $2(0)$ & $4(0)$ & $7(0)$ & $6(0)$ & $5(0)$ & $1(0)$ & $28(0)$ \\
6 & $(0)$ & $1(0)$ & $1(0)$ & $(0)$ & $1(0)$ & $(0)$ & $(0)$ & $3(0)$ \\
Total & $4,203,541(95.5)$ & $187,062(4.2)$ & $10,765(0.24)$ & $953(0)$ & $119(0)$ & $20(0)$ & $4(0)$ & $4,402,464(100)$
\end{tabular}

$A D H D$ attention-deficit hyperactivity disorder

${ }^{a}$ The figure in parentheses represents the percentage of the total number of subjects $(4,402,464)$

Table 2 Number of subjects exposed to asthma medications, with their number of prescribers and pharmacies visited ${ }^{\mathrm{a}}$

\begin{tabular}{llllllll}
\hline Number of pharmacies & 1 & 2 & 3 & 4 & 5 & 9 & Total \\
\hline Number of prescribers & & & & & & \\
1 & $5,320,404(86.8)$ & $(0)$ & $(0)$ & $(0)$ & $(0)$ & $(0)$ & $5,320,404(86.8)$ \\
2 & $650,913(10.6)$ & $106,486(1.7)$ & $2,748(0)$ & $68(0)$ & $2(0)$ & $1(0)$ & $760,218(12.4)$ \\
3 & $34,526(0.6)$ & $8,731(0.1)$ & $1,169(0)$ & $44(0)$ & $2(0)$ & $(0)$ & $44,472(0.7)$ \\
4 & $1,931(0)$ & $665(0)$ & $147(0)$ & $18(0)$ & $2(0)$ & $(0)$ & $2,763(0.1)$ \\
5 & $85(0)$ & $52(0)$ & $17(0)$ & $6(0)$ & $(0)$ & $(0)$ & $160(0)$ \\
6 & $3(0)$ & $3(0)$ & $(0)$ & $1(0)$ & $(0)$ & $(0)$ & $7(0)$ \\
7 & $(0)$ & $(0)$ & $1(0)$ & $(0)$ & $(0)$ & $(0)$ & $1(0)$ \\
Total & $6,007,862(98)$ & $115,937(1.9)$ & $4,082(0.07)$ & $137(0)$ & $6(0)$ & $1(0)$ & $6,128,025(100)$ \\
\hline
\end{tabular}

${ }^{a}$ The figure in parentheses represents the percentage of the total number of subjects $(6,128,025)$

$(0.6 \%)$ of $2,360,546$ non-naïve subjects, and in 4,423 $(0.2 \%)$ of 2,041,918 naïve subjects (Table 3$)$. Note that this prevalence of shopping behavior is higher than that observed in Table 1 because of the different ways episodes were counted, as described in Sect. 2.4.

Among subjects who shopped, the median time from the first dispensing of ADHD medications to the first shopping episode was approximately 7 months, and was slightly shorter in non-naïve subjects than naïve subjects (Table 3).

Approximately $58 \%$ of all subjects dispensed ADHD medications who exhibited shopping behavior had only one episode of shopping during the 18 months of follow-up, and these subjects accounted for $22.4 \%$ of all shopping episodes. In contrast, the $9.2 \%$ of shoppers who shopped six times or more accounted for $42.0 \%$ of all the shopping episodes (Table 4). Relative to non-shoppers and the overall group of shoppers, these latter subjects were more likely to be between 30 and 39 years of age and not naïve to ADHD medications (Table 3).

Dispensing of stimulant ADHD medications was more common among subjects exhibiting shopping behavior than among subjects without such behavior; odds ratio 8.3, $95 \%$ confidence interval 6.9-10.2 (Table 5).

\section{Discussion}

This large population-based cohort study suggests that the operational definition of ADHD medication shopping behavior has the greatest discriminative value when subjects have overlapping prescriptions for ADHD medications written by different prescribers and filled at three or more pharmacies. Similar behavior is very rare in subjects prescribed asthma medications.

A study conducted in a French claims database found, similar to our findings, that a small proportion of subjects $(0.5-1 \%)$ received their medication from a large number of distinct prescribers and pharmacies, which suggested abuse [14].

The definition of shopping behavior for ADHD medications is the same as for opioids [7, 8]. Similarly to opioid shopping behavior, shopping behavior is observed in less than $1 \%$ of those dispensed ADHD medications and tended to occur in young adults; approximately half the subjects who exhibited shopping behavior did so only once, and a small proportion of subjects accounted for a disproportionately large percentage of shopping episodes. As for opioids, subjects with prior exposure were more likely 
Table 3 Incidence of shopping behavior, time to first shopping episode, number of subjects with six or more shopping episodes, by age, sex, and prior use of ADHD medications

\begin{tabular}{|c|c|c|c|c|}
\hline Group & $\begin{array}{l}\text { Number of subjects exposed to } \\
\text { ADHD medications (col. \%) }\end{array}$ & $\begin{array}{l}\text { Number (col. \%) of subjects } \\
\text { with shopping behavior }\end{array}$ & $\begin{array}{l}\text { Number of days to first } \\
\text { shopping episode (median) }\end{array}$ & $\begin{array}{l}\text { Number (col. \%) of subjects with } \\
\text { six or more shopping episodes }\end{array}$ \\
\hline Total & $4,402,464$ & $18,130(0.4)$ & 225 & $1,666(9.2)$ \\
\hline \multicolumn{5}{|l|}{ Age, years } \\
\hline$<10$ & $640,430(14.5)$ & $2,322(12.8)$ & 287.5 & $70(4.2)$ \\
\hline $10-19$ & $1,714,153(38.9)$ & $3,794(20.9)$ & 246 & $193(11.6)$ \\
\hline $20-29$ & $743,932(16.9)$ & $4,517(24.9)$ & 227 & $418(25.1)$ \\
\hline $30-39$ & $457,853(10.4)$ & 3,789 (20.9) & 190 & $506(30.4)$ \\
\hline $40-49$ & $392,840(8.9)$ & $2,084(11.5)$ & 202.5 & $253(15.2)$ \\
\hline $50-59$ & $296,421(6.7)$ & $1,275(6)$ & 195 & $175(10.5)$ \\
\hline $60-69$ & $116,655(2.6)$ & $302(1.7)$ & 163 & $45(2.7)$ \\
\hline$\geq 70$ & $40,180(0.9)$ & $47(0.3)$ & 207 & $6(0.4)$ \\
\hline \multicolumn{5}{|l|}{ Sex } \\
\hline Female & $1,934,829(43.9)$ & 8,807 (48.6) & 214 & $910(54.6)$ \\
\hline Male & $2,467,635(56.0)$ & $9,323(51.4)$ & 234 & $756(45.4)$ \\
\hline \multicolumn{5}{|c|}{ Prior use of ADHD medications } \\
\hline Naïve & $2,041,918(46.4)$ & $4,423(24.4)$ & 237 & $222(13.3)$ \\
\hline Non-naïve & $2,360,546(53.6)$ & 13,707 (75.6) & 221 & $1,444(86.7)$ \\
\hline
\end{tabular}

Prior use of ADHD medications refers to the presence or absence of dispensing 4 months prior to the initial prescription in the study period $A D H D$ attention-deficit hyperactivity disorder, Col. Column

Table 4 Frequency of shopping episodes for subjects with shopping behavior

\begin{tabular}{lll}
\hline $\begin{array}{l}\text { Number of shopping } \\
\text { episodes during the follow-up period }\end{array}$ & $\begin{array}{l}\text { Number (\%) of subjects } \\
\text { with shopping behavior }\end{array}$ & $\begin{array}{l}\text { Number (\%) of } \\
\text { shopping episodes }\end{array}$ \\
\hline 1 & $10,413(57.4)$ & $10,413(22.4)$ \\
2 & $3,345(18.5)$ & $6,690(14.4)$ \\
3 & $1,443(8.0)$ & $4,329(9.3)$ \\
4 & $795(4.4)$ & $3,180(6.9)$ \\
5 & $468(2.6)$ & $2,340(5.0)$ \\
$6-9$ & $915(5.1)$ & $6,637(14.3)$ \\
$10-20$ & $585(3.2)$ & $7,834(16.9)$ \\
$21-83$ & $166(0.9)$ & $4,992(10.8)$ \\
Total & 18,130 & 46,415 \\
\hline
\end{tabular}

to become shoppers. Also in parallel with opioid shoppers, who tended to receive strong opioids, ADHD medication shoppers were more likely to receive ADHD medications that are stimulants.

The fact that the criteria that serve to identify subjects who engage in ADHD medication shopping behavior and opioid shopping behavior are similar seems to suggest that overlapping prescriptions written by different prescribers and filled at three or more pharmacies can be used as an operational definition to assess shopping behavior for medications that are prone to abuse and diversion in general. It is worth noting that subjects abusing a specific drug are likely to abuse other drugs or have a higher risk of developing abuse when exposed to other medications with abuse potential [15-18].

In contrast to opioids for which $0.5 \%$ of shoppers were aged 18 years or younger [8], we found that at least $13 \%$ of shoppers were very young (less than 10 years of age); this finding likely represents diversion by their parents or caregivers [19]. We also found that a small number of subjects were responsible for a disproportionately large number of shopping episodes, which likely also represents diversion of ADHD medications. A survey of undergraduate students found that their leading source of ADHD medications for non-medical use was friends and peers [20]. 
Table 5 Type of ADHD dispensed to subjects with and without shopping behavior ${ }^{\mathrm{a}}$

\begin{tabular}{lccc}
\hline & $\begin{array}{l}\text { Number (\%) of subjects } \\
\text { without shopping behavior }\end{array}$ & $\begin{array}{l}\text { Number (\%) of subjects } \\
\text { with shopping behavior }\end{array}$ & $\begin{array}{l}\text { Odds ratio (95\% CI) for shopping } \\
\text { behavior vs. being dispensed any } \\
\text { stimulant ADHD medication }\end{array}$ \\
\hline Stimulants & $4,179,353(95.3)$ & $18,024(99.4)$ & $8.3(6.9-10.2)$ \\
No stimulants & $204,981(4.7)$ & $106(0.6)$ & $18,130(100)$ \\
Total number (\%) of subjects & $4,384,334(100)$ & \\
\hline
\end{tabular}

$A D H D$ attention-deficit hyperactivity disorder, $C I$ confidence interval

${ }^{\text {a }}$ For subjects exhibiting shopping behavior, we looked for stimulants during any shopping episode; for non-shoppers we looked for stimulants during any dispensing

The low frequency of shopping behavior observed in this study is likely to be an underestimate of the true incidence. The LRx database does not have $100 \%$ coverage of all pharmacy transactions in the US, and online transactions are not covered. Of note, the matching algorithm used to uniquely identify subjects could fail to identify two subjects as the same individual if a minimum number of required encrypted attributes did not match, and thus would fail to discern a subject who presented false identification. However, no other data source will permit an assessment across the whole of the US or will capture cash prescriptions, which are very relevant when evaluating the risk of diversion [8].

We aimed for a definition that would avoid false positives (subjects who, for many reasons, could have different prescribers and pharmacies but were not shopping). A definition that limits misclassification of subjects, especially by reducing the number of false positive subjects, is crucial for research and health policy. To obtain such definition, we compared subjects dispensed asthma medications, which are less likely to be abused, with subjects dispensed ADHD medications with a higher intrinsic risk of abuse. Asthma and ADHD medications differ with respect to scheduling, and may differ in patterns of prescription (e.g. number of prescribers involved in care). These distinctions may have differentially affected our estimates of the numbers of prescribers and pharmacies visited by subjects in the asthma medication cohort and thus confounded the observed differences in shopping behavior between the two groups. In addition, this study did not address possible differences in socioeconomic status between the asthma and ADHD medication cohorts. For example, if the prevalence of asthma and lack of continuity in care are associated with low socioeconomic status, then this could lead to a higher risk of a subject with asthma being classified as a shopper, with socioeconomic status being a mediating factor.

We found a small difference in the median time to first shopping episode between naïve and non-naïve
ADHD medication subjects. The small size of this difference may reflect misclassification error, with subjects who were non-naïve being classified as naïve because the look-back period that we implemented was limited to 4 months, while the recommended medication-free period ('drug holiday') for ADHD medications may have extended beyond 4 months. We also observed dispensings of ADHD medications to subjects aged 70 years or older. These dispensings could be for the treatment of conditions different from ADHD. However, we report the incidence of shopping behavior stratified by age category.

This study did not assess the intent of subjects who engaged in shopping behavior or the association with the comorbid diagnosis of substance abuse or dependence. It can be argued that counting the number of distinct pharmacies and prescribers is more objective and accurate than measuring a construct that is subjective and difficult to measure, such as abuse or dependence. Our intent was to find a combination of pharmacies and prescribers that differentiated between subjects prescribed ADHD medications who were more likely to engage in non-medical use, and subjects prescribed medications that are not prone to abuse or dependence, such as asthma medications. We found such a definition. Furthermore, the behavior was more commonly observed in young subjects, which strengthens the validity of the findings. In addition, the definition for ADHD medication shopping behavior was found to be the same as the one used to define opioid shopping behavior, and that definition has been explicitly linked to opioid abuse [21]. Nonetheless, understanding why subjects need to visit multiple pharmacies and prescribers, and determining whether or not they are misusing, abusing, or diverting the ADHD medications, will increase the acceptance of the definition of shopping behavior as it relates to ADHD medications, and will help health care providers or insurers implement monitoring to decrease the risk of abuse or diversion. 


\section{Conclusions}

ADHD medication shopping behavior can be defined as subjects with overlapping prescriptions written by two or more prescribers and filled at three or more pharmacies. Shopping behavior is more commonly observed in younger ages, and a small number of subjects is responsible for a disproportionately large number of shopping episodes.

Declaration of interest M.S. Cepeda, D. Fife, and J. Berwaerts are employees of Janssen Research and Development, LLC, an affiliate of Janssen Pharmaceuticals, Inc. which markets CONCERTA ${ }^{\circledR}$ brand methylphenidate $\mathrm{HCl}$, an ADHD medication. They hold stocks in Johnson \& Johnson, the parent company of Janssen Research \& Development, LLC.

Open Access This article is distributed under the terms of the Creative Commons Attribution Noncommercial License which permits any noncommercial use, distribution, and reproduction in any medium, provided the original author(s) and the source are credited.

\section{References}

1. Wilens TE, Adler LA, Adams J, Sgambati S, Rotrosen J, Sawtelle $\mathrm{R}$, et al. Misuse and diversion of stimulants prescribed for ADHD: a systematic review of the literature. J Am Acad Child Adolesc Psychiatry. 2008;47(1):21-31.

2. Cassidy TA, McNaughton EC, Varughese S, Russo L, Zulueta M, Butler SF. Nonmedical use of prescription ADHD stimulant medications among adults in a substance abuse treatment population: early findings from the NAVIPPRO surveillance system. J Attend Disord. 2013 [Epub ahead of print].

3. Cassidy TA, Varughese S, Russo L, Budman SH, Eaton TA, Butler SB. Nonmedical use and diversion of ADHD stimulants among U.S. adults ages 18-49: a national Internet survey. J Attend Disord. 2012 [Epub ahead of print].

4. Arria AM, Caldeira KM, O'Grady KE, Vincent KB, Johnson EP, Wish ED. Nonmedical use of prescription stimulants among college students: associations with attention-deficit-hyperactivity disorder and polydrug use. Pharmacotherapy. 2008;28(2):156-69.

5. Substance Abuse and Mental Health Services Administration. The DAWN report. Emergency department visits involving attention deficit/hyperactivity disorder stimulant medications. Available from: http://www.samhsa.gov/data/2k13/dawn073/ sr073-add-adhd-medications.htm. Accessed 21 Oct 2013.

6. Fischer B, Bibby M, Bouchard M. The global diversion of pharmaceutical drugs non-medical use and diversion of psychotropic prescription drugs in North America: a review of sourcing routes and control measures. Addiction. 2010;105(12):2062-70.
7. Cepeda MS, Fife D, Chow W, Mastrogiovanni G, Henderson SC. Assessing opioid shopping behaviour: a large cohort study from a medication dispensing database in the US. Drug Saf. 2012;35(4):325-34.

8. Cepeda MS, Fife D, Chow W, Mastrogiovanni G, Henderson SC. Opioid shopping behavior: how often, how soon, which drugs, and what payment method. J Clin Pharmacol. 2013;53(1):112-7.

9. Cepeda MS, Fife D, Yuan Y, Mastrogiovanni G. Distance traveled and frequency of interstate opioid dispensing in opioid shoppers and nonshoppers. J Pain. 2013;14(10):1158-61.

10. Cepeda MS, Fife D, Berlin JA, Mastrogiovanni G, Yuan Y. Characteristics of prescribers whose patients shop for opioids: results from a cohort study. J Opioid Manag. 2012;8(5):285-91.

11. Wilsey BL, Fishman SM, Gilson AM, Casamalhuapa C, Baxi H, Zhang $\mathrm{H}$, et al. Profiling multiple provider prescribing of opioids, benzodiazepines, stimulants, and anorectics. Drug Alcohol Depend. 2010;112(1-2):99-106.

12. Somerford P, Katzenellenbogen J, Coddle J. Major causes of disease burden: an analysis by age. Available from: https:// wwwhealthwagovau/publications/documents/BOD/BOD5pdf. Accessed 8 Jul 2014.

13. Dickinson BD, Altman RD, Deitchman SD, Champion HC. Safety of over-the-counter inhalers for asthma: report of the council on scientific affairs. Chest. 2000;118(2):522-6.

14. Frauger E, Pauly V, Natali F, Pradel V, Reggio P, Coudert H, et al. Patterns of methylphenidate use and assessment of its abuse and diversion in two French administrative areas using a proxy of deviant behaviour determined from a reimbursement database: main trends from 2005 to 2008. CNS Drugs. 2011;25(5):415-24.

15. Lee SS, Humphreys KL, Flory K, Liu R, Glass K. Prospective association of childhood attention-deficit/hyperactivity disorder (ADHD) and substance use and abuse/dependence: a meta-analytic review. Clin Psychol Rev. 2011;31(3):328-41.

16. Turk DC, Swanson KS, Gatchel RJ. Predicting opioid misuse by chronic pain patients: a systematic review and literature synthesis. Clin J Pain. 2008;24(6):497-508.

17. Michna E, Ross EL, Hynes WL, Nedeljkovic SS, Soumekh S, Janfaza D, et al. Predicting aberrant drug behavior in patients treated for chronic pain: importance of abuse history. J Pain Symptom Manag. 2004;28(3):250-8.

18. Fleming MF, Balousek SL, Klessig CL, Mundt MP, Brown DD. Substance use disorders in a primary care sample receiving daily opioid therapy. J Pain. 2007;8(7):573-82.

19. Evans C, Blackburn D, Butt P, Dattani D. Use and abuse of methylphenidate in attention-deficit/hyperactivity disorder. Beware of legitimate prescriptions being diverted. CPJ/RPC. 2004;137(6):30-5.

20. McCabe SE, Teter CJ, Boyd CJ. Medical use, illicit use and diversion of prescription stimulant medication. J Psychoactive Drugs. 2006;38(1):43-56.

21. Cepeda MS, Fife D, Kihm MA, Mastrogiovanni G, Yuan Y. Comparison of the risks of shopping behavior and opioid abuse between tapentadol and oxycodone and association of shopping behavior and opioid abuse. Clin J Pain. 2013 [Epub ahead of print]. 